\title{
Energy harvesting from suspension system using regenerative force actuators
}

\section{Farbod Khoshnoud}

School of Engineering and Technology,

University of Hertfordshire,

College Lane Campus, Hatfield, AL10 9AB, UK

Email: f.khoshnoud@herts.ac.uk

Industrial Automation Laboratory,

Department of Mechanical, Engineering,

The University of British Columbia,

Vancouver, BC, V6T 1Z4, Canada

*Corresponding author

\section{Dinesh B. Sundar}

School of Engineering and Technology, University of Hertfordshire,

College Lane Campus, Hatfield, AL10 9AB, UK

Email: d.sundar@herts.ac.uk

Nuri M. Badi

School of Engineering and Technology,

University of Hertfordshire,

College Lane Campus, Hatfield, AL10 9AB, UK

Email: N.Badi@herts.ac.uk

\section{Yong K. Chen}

School of Engineering and Technology,

University of Hertfordshire,

College Lane Campus, Hatfield, AL10 9AB, UK

Email: y.k.chen@herts.ac.uk

\section{Rajnish K. Calay}

School of Engineering and Technology,

University of Hertfordshire,

College Lane Campus, Hatfield, AL10 9AB, UK 
Author

Email: r.k.calay@herts.ac.uk

\title{
Clarence W. de Silva
}

Department of Mechanical, Engineering,

The University of British Columbia,

Vancouver, BC, V6T 1Z4, Canada

Email: desilva@mech.ubc.ca

\begin{abstract}
In this paper harvesting vibration energy from suspension is investigated. Theoretical values for the harvested energy are calculated. Experimental evaluation of the energy is performed using vehicle road simulation facilities. An excitation signal in the frequency range of $0.5 \mathrm{~Hz}$ to $20 \mathrm{~Hz}$ is applied to the vehicle and the harvested power is calculated. Experimental results give a maximum harvested power of $984.4 \mathrm{~W}$ at the highest frequency, which is close to the theoretically computed value of $1106 \mathrm{~W}$, for each suspension. Application of Regenerative Force Actuators (RFA) is explored for harvesting the vibration energy and controlling vibration. It is shown that the harvested power increases with the value of the actuator constant.
\end{abstract}

Keywords: Energy harvesting from vibration; Regenerative Force Actuators; Active Control of Suspensions.

\section{Introduction}

Dissipation of energy by shock absorbers in vehicle suspensions is a main sources of energy loss. Only $10 \%$ to $16 \%$ of the fuel energy is used to drive a car (Zuo, L. et al., 2010). A regenerative shock absorber can harvest $16 \mathrm{~W}$ to $64 \mathrm{~W}$ of power (at $0.25 \mathrm{~m} / \mathrm{s}$ to $0.5 \mathrm{~m} / \mathrm{s}$ RMS suspension velocity). More recent research (Zuo, L. et al., 2012) shows that about $100 \mathrm{~W}$ to $400 \mathrm{~W}$ power is available from suspension at $26.8 \mathrm{~m} / \mathrm{s}$ vehicle speed. It is shown that significant amount of the energy, about $20 \%$ to $70 \%$ of the power required a typical vehicle to travel at 20 $\mathrm{m} / \mathrm{s}$, can be recovered and stored (Goldner R. B., et al., 2001).

Regenerative Force Actuators (RFA) (Scruggs J.T., and Skelton, R.E., et al. 2006, and Scruggs, J.T., 2004) can harvest energy from vibration and use that energy for control of car suspension. Regenerative actuators which are composed of linear DC motors can provide better vibration isolation than passive and semiactive systems and they do not consume any energy (Suda, Y. et al., 1998). A 
self-powered active suspension controls vibration without external energy for various applications such as suspension or truck cabins (Nakano, K. and Suda., Y., 2004). A regenerative self-powered active vibration control can produce more energy than it consumes (Nakano K., et al. 2003). A regenerative actuator can produce more energy at higher speeds and an active control system can utilize the generated energy for high speed and low speed vibration control (Okada, Y. et al., 1997). At low speeds a regenerative actuator may not generate sufficient energy to control the vibration efficiently. Designing a control technique to regenerate energy from low speed and low voltage actuator to high voltage charging circuit can overcome this problem, where the damping coefficient can be manipulated (Kim, S. S., and Okada, Y., 2002). An electric shock absorber can store part of the generated energy in the automobile battery (Paz, O. D., 2004). Electromagnetic regenerative dampers can be designed as rotating or linear dampers. Rotating electromagnetic dampers can provide mechanical amplification, but degrade the performance of vehicle dynamics. The performance can be improved by adding dynamic elements in series with the rotating damper (Graves, K. E. et al., 2000). A regenerative actuator can have up to $70 \mathrm{~kg}$ weight which is not suitable for a passenger vehicle (Goldner, R. B., 2005) or bulky (Gupta, A. et al., 2006). Application of rare-earth permanent magnets and high permeable magnetic loops to configure a four-phase linear generator can increased efficiency and lower the weight of a regenerative actuator (Zuo, L. et al., 2010).

Quarter, half and full car models are developed for analysing dynamics of the suspension and control systems of a vehicle where responses of these models generally give similar results (Faris, W. F. et al., 2009). Suspension control techniques include passive, semi-active and active control systems. Typical control policies, namely, skyhook, ground-hook and hybrid controls, are applicable in semi-active systems suspension control. The hybrid control policy can provide a better compromise between comfort, road-holding and suspension displacement than the skyhook and ground-hook control techniques (Ihsan, S. I., et al., 2007, and Faris, W. F., et al., 2009). Various other techniques have been developed in control of vehicles suspension. For instance, a Non-linear Energy Sink (NES) controller can control the dynamics of vehicle suspension systems actively or semi-actively. This system utilises non-linear spring and damping elements which can improve the handling performance, absorb vibration energy transmitted by road disturbance and improve the vehicle ride comfort, with a balance between ride comfort and handling performance (Zheng, L. and Baz, A.). 
The above introduction regarding regenerative actuators represents the importance of such device is saving fuel in a vehicle. In the research in this paper the amount of energy that can be harvested from suspension is investigated using a road simulation rig and the experiment is performed on an actual car with an experimental setup of estimating the amount of harvested energy for a wide range of frequency excitations. Energy harvesting from suspension using regenerative actuators is explored. Theoretical analysis of harvested power form suspension is performed. Experimental investigation of power generation is explored using a road simulator rig on a Ford Focus vehicle. Effect of regenerative actuators on harvesting power is studied.

\section{Energy harvesting and vehicle dynamics}

A single degree of freedom quarter model of a car is illustrated in Figure 1. This is a mass-spring-damper model of the suspension system. The dynamic behaviour of this system is studied in order to investigate the amount of energy that can be harvested from the suspension vibration. In practice, if the damper of a suspension system is replaced with a Regenerative Force Actuator (RFA) then the regenerative system can convert the vibration energy to useful electrical energy. The harvested electrical energy can be stored and/or used to control the same vibration and provide ride comfort for the passengers. The stored electrical energy can provide partial power in hybrid vehicles. The effect of tyre vibrations is not considered in this model as it does not play any significant role in energy harvesting.

Figure 1 Dynamic model of the suspension system of a quarter car model and representation of a regenerative actuator for energy harvesting applications.

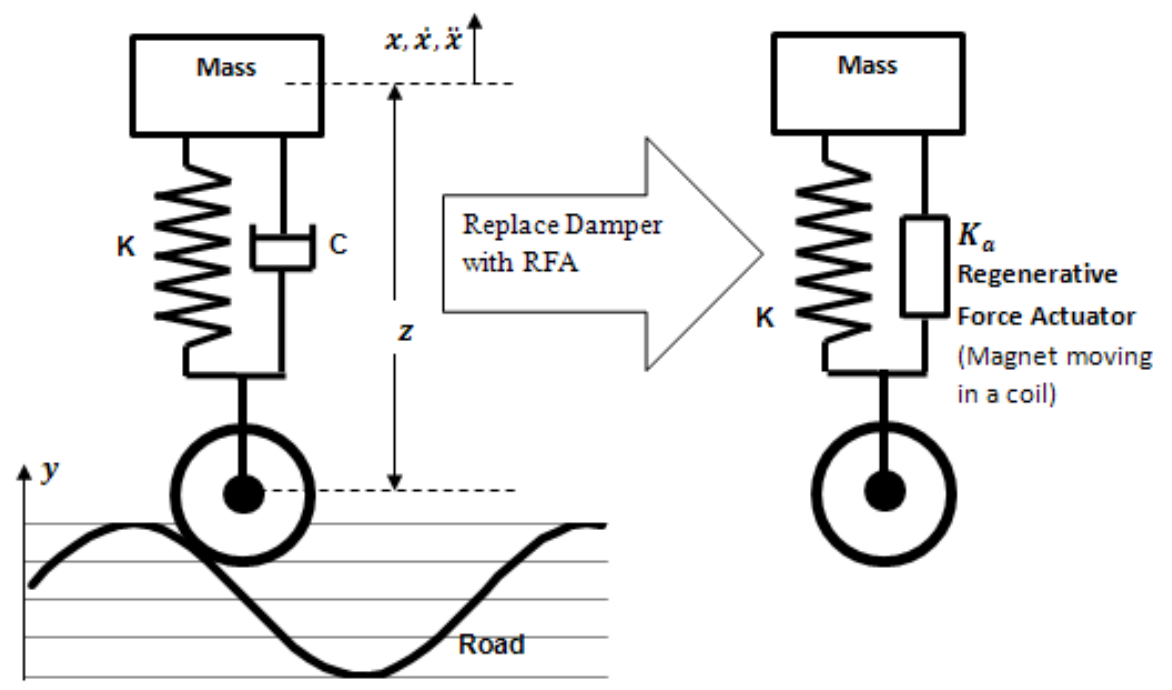


The equation of motion of the quarter car model is given by (De Silva, C. W., 2007)

$$
m \ddot{x}+c(\dot{x}-\dot{y})+k(x-y)=0
$$

where $y$ denotes the road profile and $x$ is the mass displacement. If $z=x-y$ is the displacement of the wheel relative to the mass then rewriting the equation in terms of $z$ gives

$$
m \ddot{z}+c \dot{z}+k z=m \ddot{y}
$$

For a harmonic excitation of $y=Y \sin \omega t$ the above equation is obtained as

$$
m \ddot{z}+c \dot{z}+k z=m \omega^{2} Y \sin \omega t
$$

and the steady state solution can be considered as

From Equations (3) and (4) we have

$$
z=Z \sin (\omega t-\phi)
$$

$$
Z=\frac{m \omega^{2} Y}{\sqrt{\left(k-\omega^{2} m\right)^{2}+c^{2} \omega^{2}}}
$$

If the dissipated energy from the damper can be harvested through a regenerative mechanism then the harvested power (Khoshnoud F., and de Silva, C. W., 2012, and Khoshnoud F., et, al. 2011) can be obtained as

$$
P=c \dot{Z} \times \dot{Z}
$$

From Equation (4), $\dot{z}=\omega Z \cos (\omega t-\phi)$, and therefore the power is determined as

$$
P=c \omega^{2} Z^{2} \cos ^{2}(\omega t-\phi)
$$

In one cycle with period of $\tau=2 \pi / \omega$ the energy is determined by

$$
E=c \omega^{2} Z^{2} \int_{0}^{2 \pi / \omega} \cos ^{2}(\omega t-\phi) \mathrm{dt}=\pi c \omega Z^{2}
$$

Hence the average power can be calculated as

$$
P=E / \tau=c \omega^{2} Z^{2} / 2
$$

From Equations (5) and (9) the power is obtained as

$$
P=\frac{c m^{2} \omega^{6} Y^{2}}{2\left(\left(k-\omega^{2} m\right)^{2}+c^{2} \omega^{2}\right)}
$$

From Equation (5) maximum road deflection $\left(Y_{\max }\right)$ can be presented as

$$
Y_{\text {max }}=\frac{\sqrt{\left(k-\omega^{2} m\right)^{2}+c^{2} \omega^{2}} Z_{\text {max }}}{m \omega^{2}}
$$

Consequently the power in Equation (10) can be rewritten for $Y_{\max }$ as

$$
P=\frac{c m \omega^{4} Y Z_{\text {max }}}{2 \sqrt{\left(k-\omega^{2} m\right)^{2}+c^{2} \omega^{2}}}
$$

The dimensionless power (Stephen, 2006) can be considered as

$$
P_{d}=\frac{P}{m \omega^{3} Y Z_{\max }}=\frac{\zeta \cdot \eta}{\sqrt{\left(1-\eta^{2}\right)^{2}+(2 \zeta \eta)^{2}}}
$$

where $\zeta$ denotes the damping ratio and $\eta$ is the frequency ratio, and $\frac{c}{m}=2 \zeta \omega_{n}$ and $\eta=\frac{\omega}{\omega_{n}}$.

The dimensionless power $P_{d}$ is plotted versus the damping ratio $\zeta$ and the frequency ratio $\eta$ in Figure 2. 
Figure 2 Dimensionless power versus frequency ratio and damping ratio.

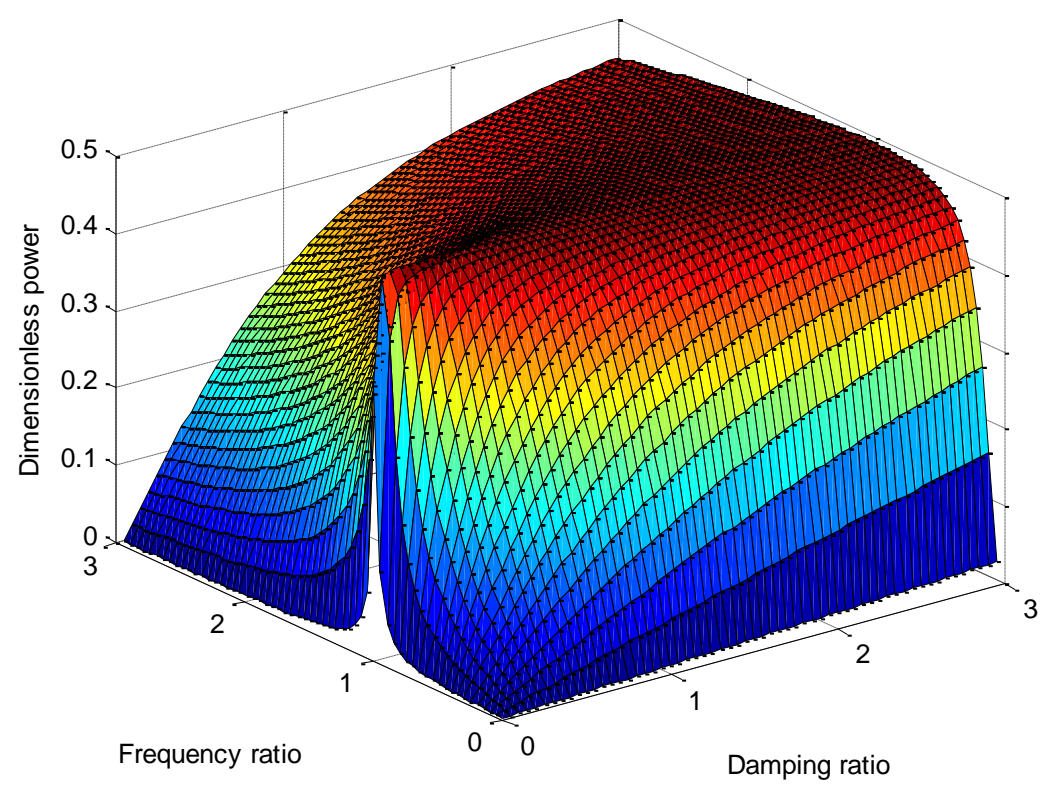

The dimensionless power $P_{d}$ in Figure 2 represents the behaviour of general vibratory systems which the maximum response occurs at frequency ratio $\eta=1$ or when frequency of excitation is equal to the natural frequency of the system. It also represents the effect of damping in the harvested power. As mentioned earlier the value for damping ratio in this simulation is equivalent to a value that corresponds to a constant of the RFA system. The actuator constant will be discussed further in next sections. As shown in Figure 2, larger values of damping ratio (or RFA constant) give higher power magnitudes. The dimensionless power does not demonstrate the effect of vehicle velocity on power magnitudes. Therefore the actual power value is investigated further using Equation (13) as below

$$
P=\frac{m \omega^{3} Y Z_{\max } \zeta \cdot \eta}{\sqrt{\left(1-\eta^{2}\right)^{2}+(2 \zeta \eta)^{2}}}
$$

The power $P$ in Equation (14) is plotted versus the damping ratio $\zeta$ and the frequency $f$ in Figure 3, where $f=\frac{\omega}{2 \pi}$. Based on this figure, it is observed that higher power is harvested for larger values of frequency $f$, where the power is proportional to the cube of the frequency. The same result as in Figure 2 is valid here regarding damping ratio $\zeta$ where larger values of damping ratio (or RFA constant) give higher power magnitudes. The two important outcomes from the power analysis include: a) exciting the suspension system with a frequency equal to its natural frequency generates higher power values (Figure 2), b) the power is proportional to the cube of the excitation frequency (Figure 3). 
Figure 3 Harvested power versus frequency and damping ratio.

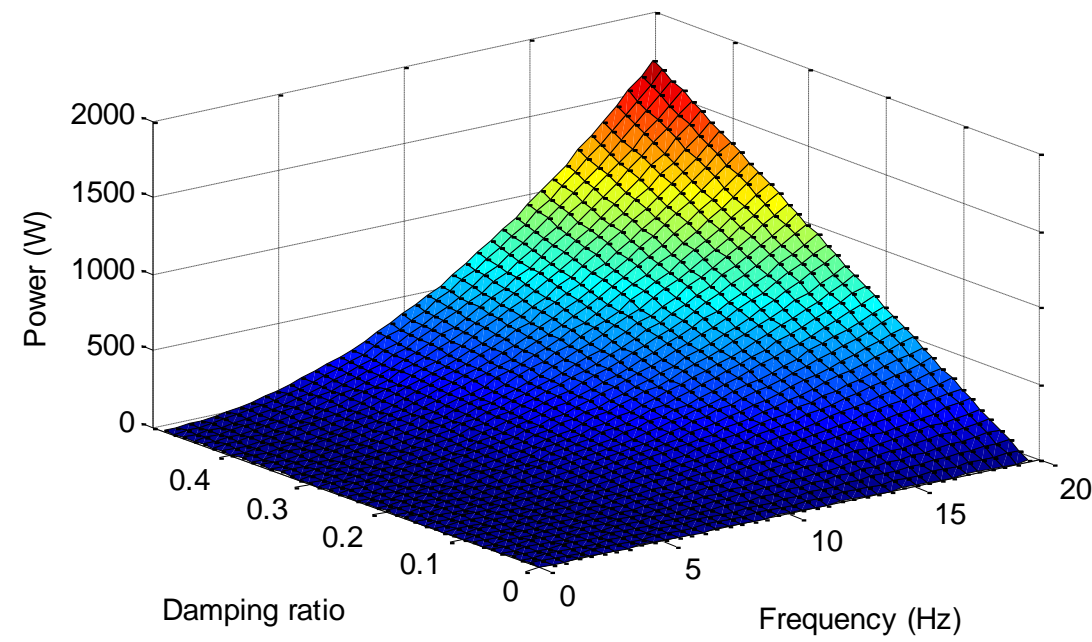

For a particular value of damping ratio $\zeta=0.3$, the power $P$ in Equation (14) is plotted versus frequency in Figure 4 . In this calculation, $\omega_{n}=\sqrt{\mathrm{k} / \mathrm{m}}=7.56$ $\mathrm{rad} / \mathrm{s}$, where the stiffness $k$ is $16 \mathrm{kN} / \mathrm{m}$ and the mass $m$ is $280 \mathrm{~kg}$. This figure (same as in Figure 3) illustrates that the maximum power is harvested at the highest frequency of $f=20 \mathrm{~Hz}$. The power at $20 \mathrm{~Hz}$ is equal to $P=1106 \mathrm{~W}$.

Figure 4 Harvested power versus frequency for damping ratio $=0.3$.

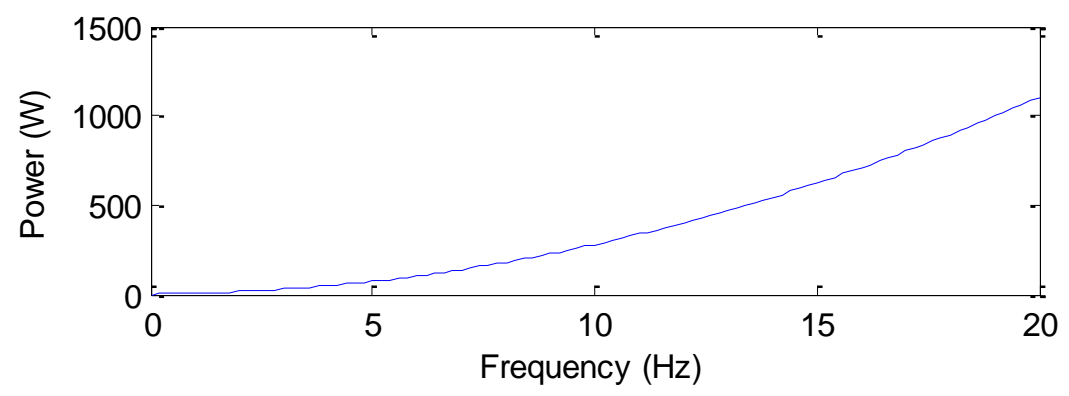

3 Experimental investigation of the harvested energy

Experimental evaluation of the harvested energy is carried out for a suspended vehicle body and unsuspended wheels in the vertical plane using the road simulator rig in Figure 5. This figure illustrates the front and rear setups for accelerometers, LVDTs (Linear variable differential transformer) and shakers. Figure 6 demonstrates the experimental setup for front left and front right of the vehicle. 
Figure 5 Road simulator rig: suspended vehicle body and unsuspended wheels in the vertical plane; placement of accelerometers and LVDTs and the shaker setup for front and rear.

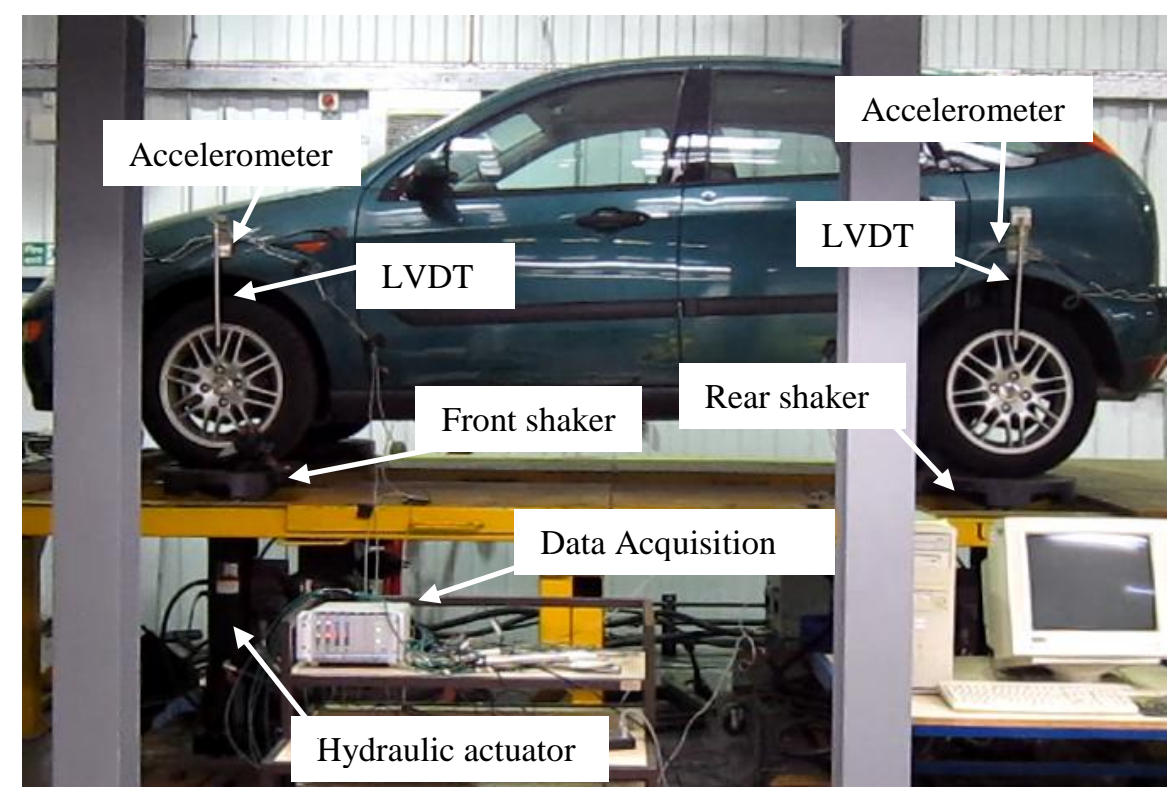

Figure 6 Demonstration of the car front experimental setup for accelerometers, LVDTs, shakers.

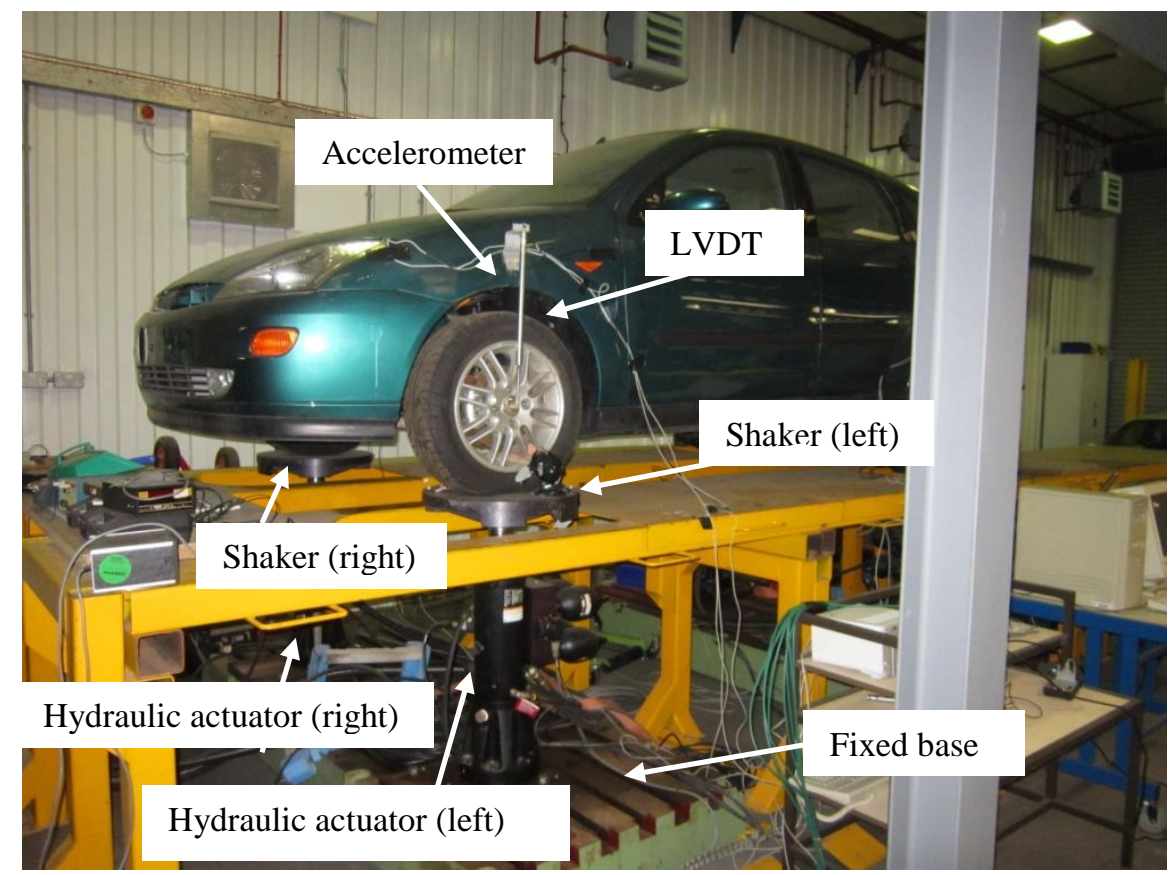


In this experiment all four wheels are excited to bounce mode of the vehicle in phase. Body acceleration and suspension displacement for the front and rear corners are measured by accelerometers and LVDTs. Sweep or increment frequency of $0.5 \mathrm{~Hz}$ to $20 \mathrm{~Hz}$ is applied to the tyres by the shakers in 40 seconds duration. The experimental data for this test is as follows. The vehicle model is a Ford Focus with total mass of $\mathrm{M}=1120 \mathrm{~kg}$, Pitch Moment of Inertia of M about $\mathrm{CG}, \mathrm{I}=1720 \mathrm{~kg} \cdot \mathrm{m}^{2}$, front suspension stiffness, $\mathrm{K}_{1}=16 \mathrm{kN} / \mathrm{m}$, and rear suspension stiffness, $\quad K_{2}=20 \mathrm{kN} / \mathrm{m}$. The LVDTs have a calibration factor of $150 \mathrm{mV} / \mathrm{mm}$. The Peak to Peak input excitation displacement is $10 \mathrm{~mm}$. Data is collected in each 4.882813e-003 seconds. Displacement of the tiers relative to mass, which is $z$ in Figure 1 or in Equation (2), is obtained from LVDT data. Variation of $z$ values versus time is given in Figure 7 for the front wheel, and in Figure 8 for the rear wheel for input excitation frequency sweep of $0.5 \mathrm{~Hz}$ to 20 $\mathrm{Hz}$ applied by the shakers to the tyres in 40 seconds duration. The peak to peak input displacement excitation, which is $Y_{\max }$ in Equation (11), is $10 \mathrm{~mm}$ in this experiment.

Figure 7 Relative displacement $\mathbf{z}$ of the left front wheel for peak to peak input displacement of $10 \mathrm{~mm}$ applied by the shaker for $0.5 \mathrm{~Hz}$ to $20 \mathrm{~Hz}$ frequency sweep in 40 seconds duration time.

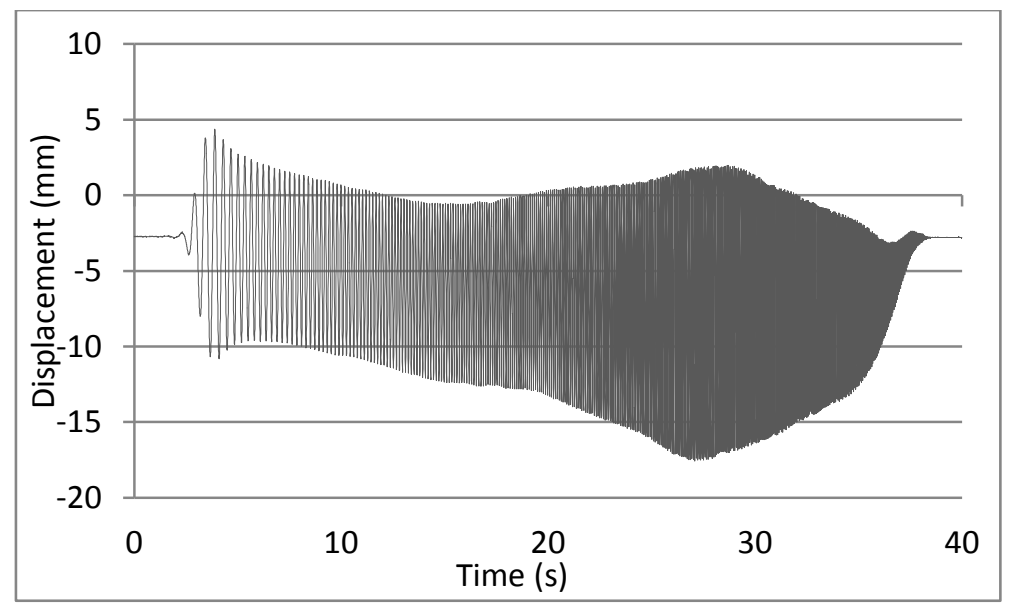

Figure 8 Relative displacement $\mathbf{z}$ of the right rear wheel for peak to peak input displacement of $10 \mathrm{~mm}$ applied by the shaker with $0.5 \mathrm{~Hz}$ to $20 \mathrm{~Hz}$ frequency sweep in 40 seconds duration time. 
Author

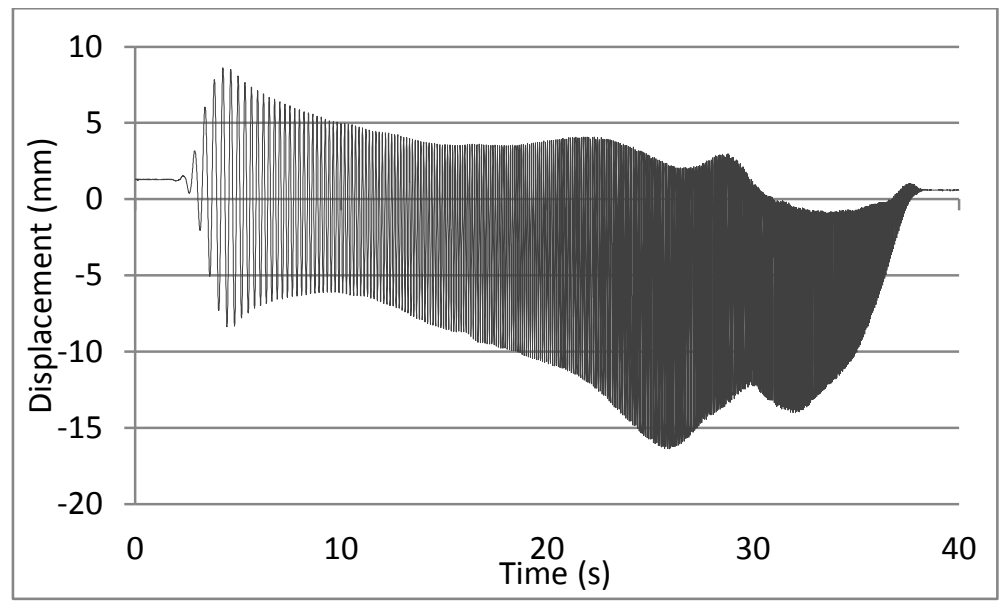

In order to illustrate the relative displacements $z$, which is shown in the above figures, with a better resolution, Figure 9 and Figure 10 represent $z$ values in a shorter time period of 8 seconds for the left front wheel and time period of 10 seconds for the right rear wheel, respectively.

Figure 9 Relative displacement $\mathbf{z}$ of the left front wheel for peak to peak input displacement of $10 \mathrm{~mm}$ applied by the shaker from 1.5 to 8 seconds.

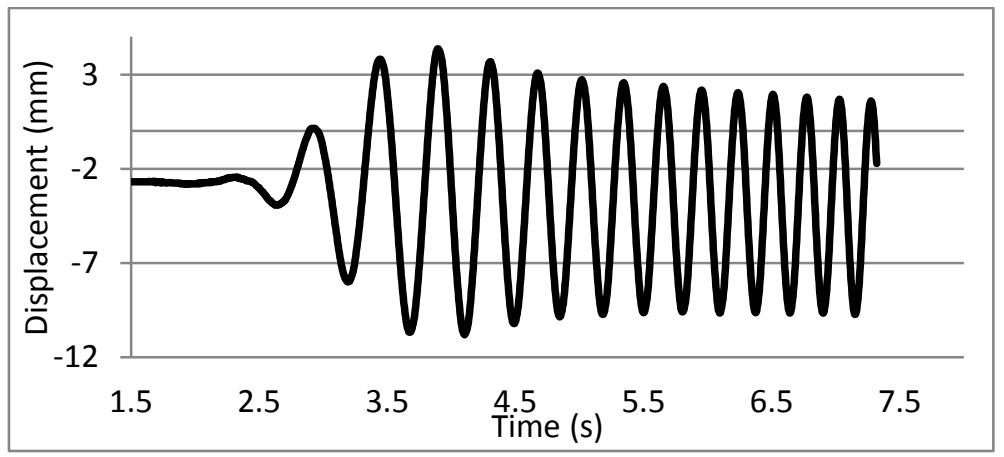

Figure 10 Relative displacement $\boldsymbol{z}$ of the right rear wheel for peak to peak input displacement of $10 \mathrm{~mm}$ applied by the shaker from 1.5 to 10 seconds. 
Title

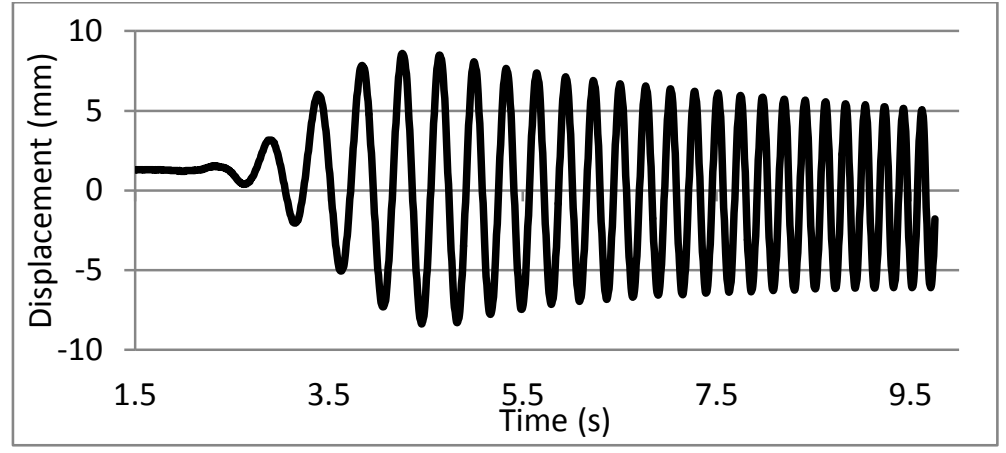

Natural frequency and damping ratio of the suspension system can be obtained from Fourier transform of the displacement data in time domain. The Fourier transform of the displacement data for the front wheel in Figure 7 is calculated. The frequency response result is presented in Figure 11.

Figure 11 Fourier transform.

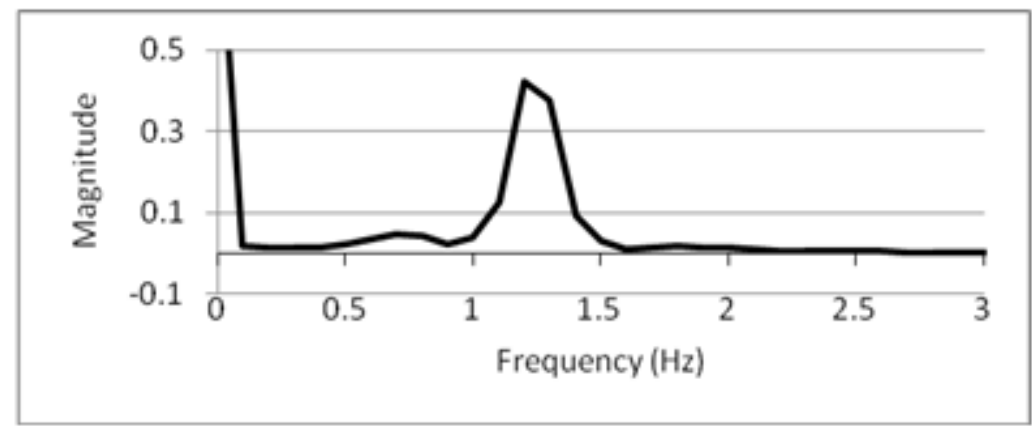

The natural frequency of the system obtained from the result in the frequency domain in Figure 11 is $1.3 \mathrm{~Hz}$ or $8.17 \mathrm{rad} / \mathrm{s}$. This is the first natural frequency of the whole car. This frequency also could be estimated from stiffness and mass values based on the quarter car stiffness and mass values as follow. For the front wheel $\omega_{n}=\sqrt{k / m}=7.56 \mathrm{rad} / \mathrm{s}$, where the stiffness $k$ is $16 \mathrm{kN} / \mathrm{m}$ and the mass $m$ is a quarter value of the total mass equal to $280 \mathrm{~kg}$. For the rear wheel $\omega_{n}=$ $\sqrt{k / m}=8.45 \mathrm{rad} / \mathrm{s}$, where the stiffness $k$ is $20 \mathrm{kN} / \mathrm{m}$ and the mass $m$ is the quarter value of the total mass equal to $280 \mathrm{~kg}$. Therefore the average calculated natural frequency is $\omega_{n}=8 \mathrm{rad} / \mathrm{s}$ which is close to the experimentally obtained natural frequency above which is $8.17 \mathrm{rad} / \mathrm{s}$.

Damping ratio of the suspension system can also be determined from the frequency response using the relation below.

$$
\zeta=\frac{\omega_{1}^{2}-\omega_{2}^{2}}{\omega_{n}^{2}}
$$


where $\omega_{1}$ and $\omega_{2}$ are the frequencies that correspond to the $1 / \sqrt{2}$ peak frequency value before and after natural frequency $\omega_{n}$ in Figure 11. Hence the damping ratio is determined as $\zeta=0.3$.

According to Equation (6) the power harvested from the vibration of a suspension system, for instance the front left suspension, can be obtained by

$$
P=c \dot{z} \times \dot{z}=2 \zeta m \omega_{n} \dot{z}^{2}
$$

As calculated above $\zeta=0.3, \omega_{n}=7.56 \mathrm{rad} / \mathrm{s}$ and the velocity $\dot{z}$ can be calculated from the experimentally measured $z$ values, where $\dot{z}=\frac{z_{n}-z_{n-1}}{t-t_{n-1}}$ and $z_{n}$ is the relative displacement value at time $t_{n}$. For instance, $\dot{z}$ can be calculated for the front left wheel associated with the 0.5 to $20 \mathrm{~Hz}$ frequency sweep excitation, and $10 \mathrm{~mm}$ peak to peak excitation displacement amplitude in Figure 7. The corresponding power values is calculated for the front left wheel based on Equation (16). The power values versus frequency are represented in Figure 12.

Figure 12 Calculation of power for experimental data.

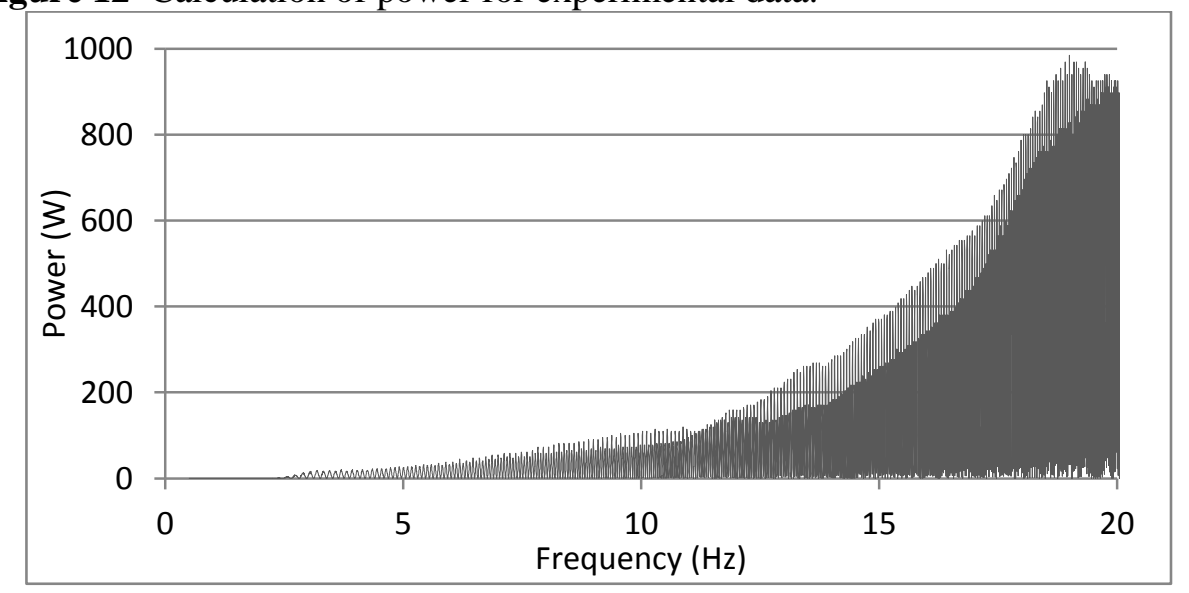

Figure 12 shows that the higher power values are generated at larger excitation frequencies. The maximum power for this result is equal to $984.4 \mathrm{~W}$ at $20 \mathrm{~Hz}$. This result is in agreement with the computational analysis result of power in the previous section which was calculated as $1106 \mathrm{~W}$ in Figure 4. At the end of the frequency sweep around $20 \mathrm{~Hz}$ the power values in Figure 12 are slightly flattened. This is only due to some experimentally inaccurate measurement when the excitation frequency is slowing down at the end of the experiment in order to return to zero value and stop. The plot in Figure 12 shows a solid filled area under the power curve. This is due to the measurement of power associated with every velocity data $\dot{z}_{n}$ for every time value $t_{n}$. The computational result in Figure 4 is illustrated for $Z_{\max }$ values and amplitude of the displacement excitation $Y$ and therefore only represents the amplitude of the power and does not give the solid filled area under the curve, which is observed in Figure 12. 
Title

\section{$4 \quad$ Regenerative actuator}

Extracting energy from the suspension system and converting vibration energy to electrical energy requires a regenerative system. Therefore the damper in the car suspension is replaced with a RFA. In order to distinguish the damper with the regenerative actuator, parameter $K_{a}$ is defined which is called actuator constant in this paper. In this paper we start by setting the values of $K_{a}$ to

$$
K_{a}=c / m
$$

Therefore this $K_{a}$ initially represents a similar dynamic behaviour as the damper. Then $K_{a}$ value is varied to investigate the effect of $K_{a}$ on the amount of harvested power.

Influence of RFA on dynamic behaviour of the suspension system is investigated below. Laplace transform of Equation (2) is

$$
s^{2} z(s)+\frac{c}{m} s z(s)+\frac{k}{m} z(s)=s^{2} y(s)
$$

The transfer function for this equation is considered as

$$
\frac{z(s)}{y(s)}=\frac{s^{2}}{\left(s^{2}+\frac{c}{m} s+\frac{k}{m}\right)}
$$

The Nyquist Plot of the above transfer function is presented in Figure 13, when $c / m=2 \zeta \omega_{n}=2 \times 0.3 \times \omega_{n}=K_{a}$, using MATLAB. The Nyquist plot shows stable closed loop system for the transfer function in Equation (19) as the gain is less than -1 for phase angle of $180^{\circ}$.

Figure 13 Nyquist Plot for the transfer function in Equation (19). 


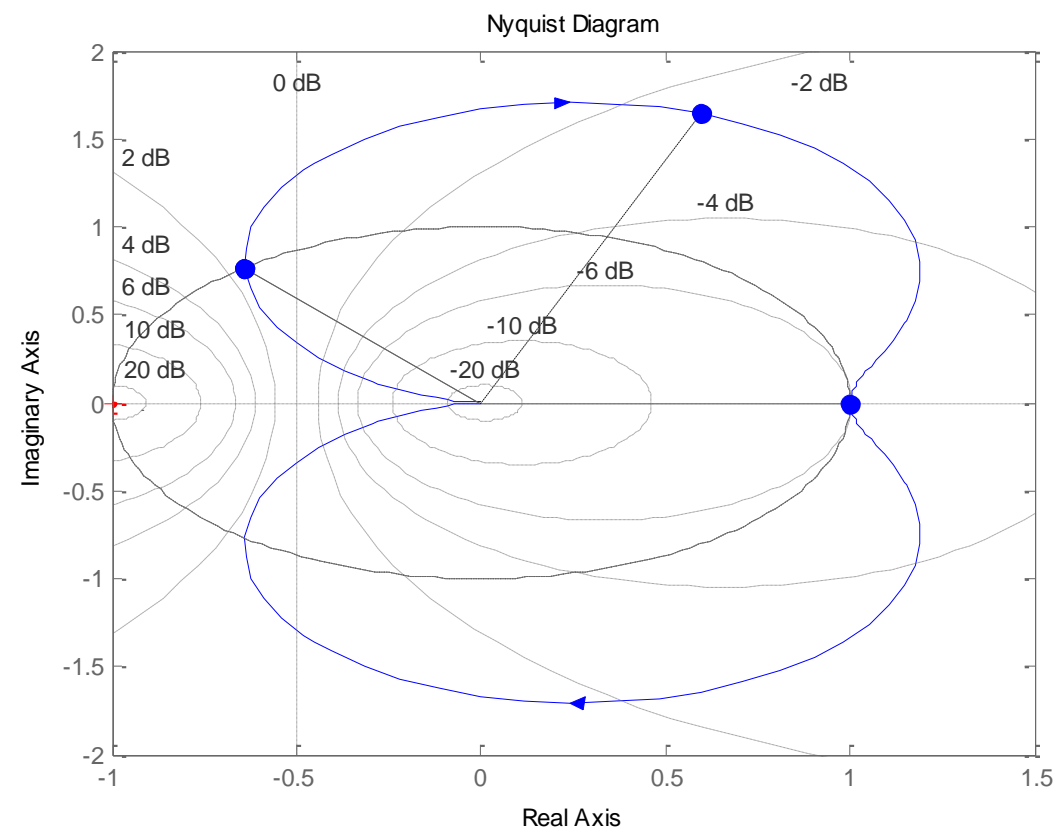

Gain $R$ and Phase Angle $\Phi$ of the transfer function are discussed below. For $\omega=\omega_{n}$ and when $s$ is $j \omega$ the gain is determined as

$$
R=\left|\frac{-\omega^{2}}{\left(\omega_{n}^{2}-\omega^{2}+2 \zeta \omega_{n} j \omega\right)}\right|=\frac{1}{2 \zeta}
$$

Therefore for $\zeta=0.3$ the Gain is $R=1.667$ (abs) or

$$
G=20 \log _{10} R=4.437 \mathrm{~dB}
$$

and the phase angle is obtained as

$$
\begin{gathered}
\phi=<\left(-\omega^{2}\right)-<\left(\omega_{n}^{2}-\omega^{2}+2 \zeta \omega_{n} j \omega\right) \\
\phi=-\tan ^{-1}\left(\frac{2 \zeta}{0}\right)=\frac{\pi}{2}
\end{gathered}
$$

For $\omega=\omega_{1}=\frac{\omega_{n}}{2}$, the gain $R_{1}=0.309$ (abs) or $G_{1}=-10.187 \mathrm{~dB}$, and $\phi=-0.38$ rad. For $\omega=\omega_{2}=2 \omega_{n}$, the gain $R_{2}=1.238(a b s)$ or $G_{2}=1.854 \mathrm{~dB}$, and $\phi=0.49 \mathrm{rad}$. Based on the above, it is observed that, as we expected, the response of the transfer function is maximum at natural frequency $\omega=\omega_{n}$.

The Gain R and phase angle $\Phi$ are plotted in Figure 14 when $c / m=2 \zeta \omega_{n}=2 \times$ $0.3 \times \omega_{n}=K_{a}$, using MATLAB Bode Plot. Both plots represent the response of the system in Equation (19) for a wide range of frequencies.

Figure 14 Bode Plot for the transfer function in Equation (19). 


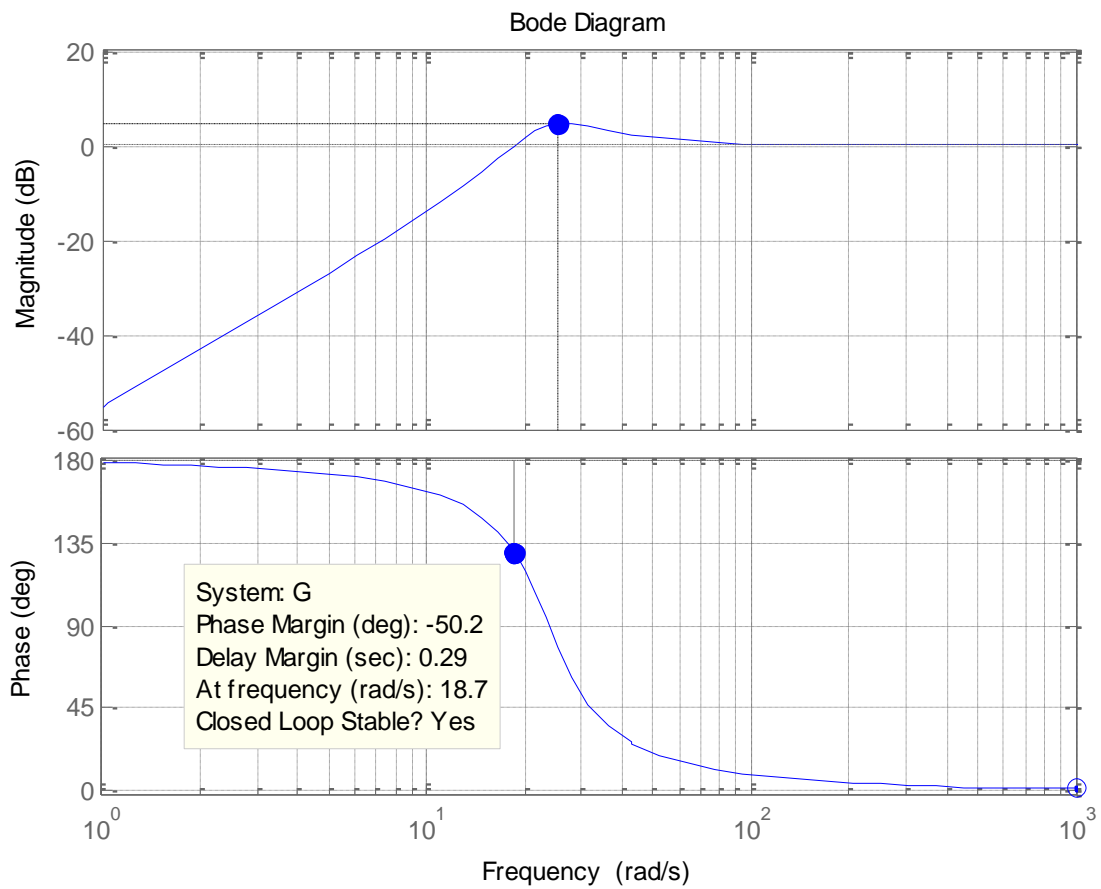

The equation of the motion of the system in (2) or the transfer function in Equation (18) can be represented by the Block diagram in Figure 15, using MATLAB SIMULINK. This block diagram can be used to obtain the Amplitude of Power $(P)$ harvested by the RFA for various excitation frequencies $\omega$ (as the input frequency of the sine wave in Figure 15). In order to obtain the amplitude of power, the input sine wave frequency is set to $\omega_{1}=\omega_{n} / 2$. This represents the input excitation frequency to the suspension system. According to Equations (16) and (17), the power generated from the vibration is obtained by

$$
P=c \dot{z} \times \dot{z}=2 \zeta m \omega_{n} \dot{z}^{2}=K_{a} m \dot{z}^{2}
$$

The block diagram in Figure 15 is constructed to determine this power value by multiplying $K_{a}$ by $\dot{z}^{2}$, as seen in the figure.

Figure 15 The block diagram for calculating the harvested power for various regenerative actuator constants. 


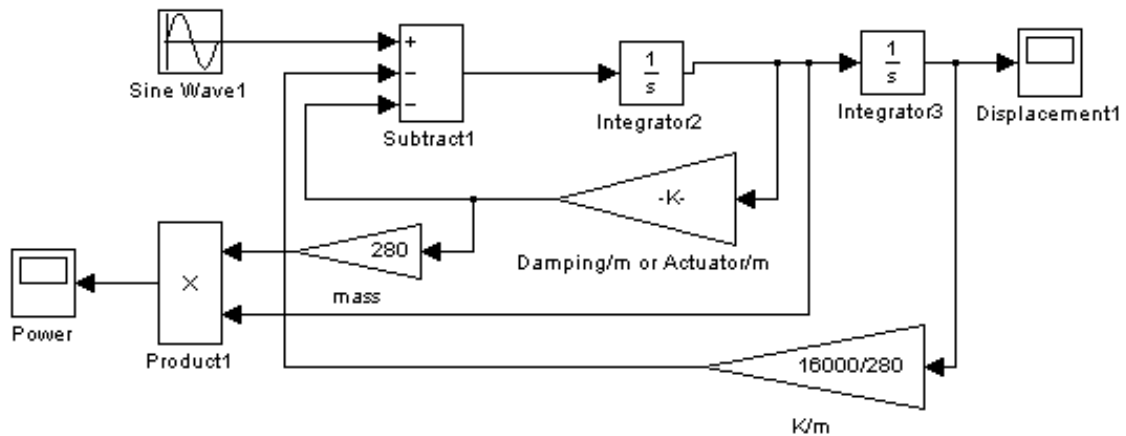

The harvested power by the RFA is obtained for actuator constant values of $K_{a}$ / $2, K_{a}, 2 K_{a}$ and $5 K_{a}$. For this experiment the value of $K_{a}$ is considered as $K_{a}=2 \zeta \omega_{n}=2 \times 0.3 \times 7.56=4.54 \mathrm{rad} / \mathrm{s}$. Initially, it is assumed that the actuator constant has the same value as the damping constant. Then this constant value is varied to $K_{a} / 2,2 K_{a}$ and $5 K_{a}$ in order to investigate the effect of actuator constant on the harvested power $P$. The harvested power for various values of actuator constants is given in Figure 16.

Figure 16 Harvested Power for various actuator constant values $K_{a}$, (a) $K_{a} / 2$, (b) $K_{a}$, (c) $2 K_{a}$, and (d) $5 K_{a}$.

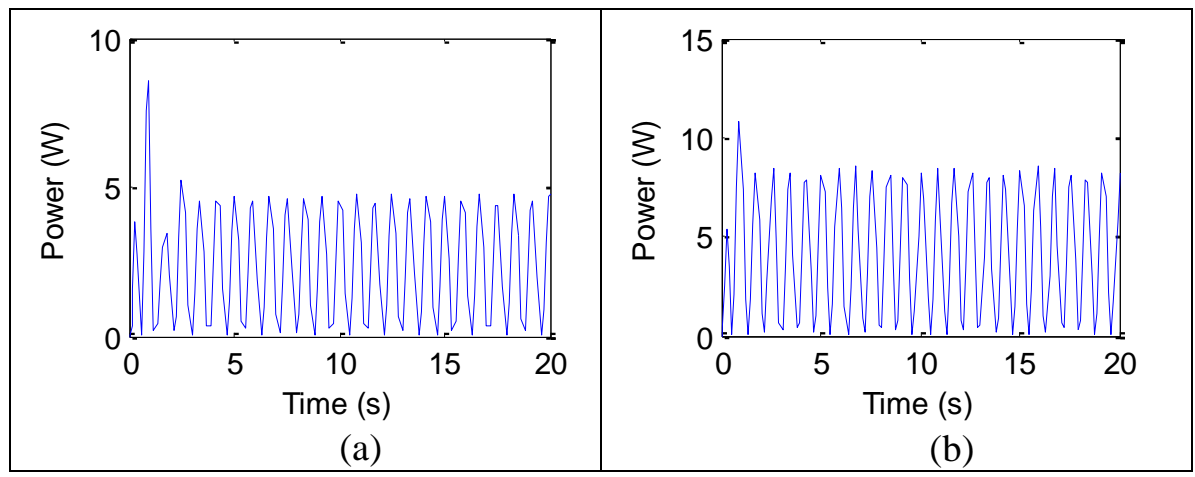




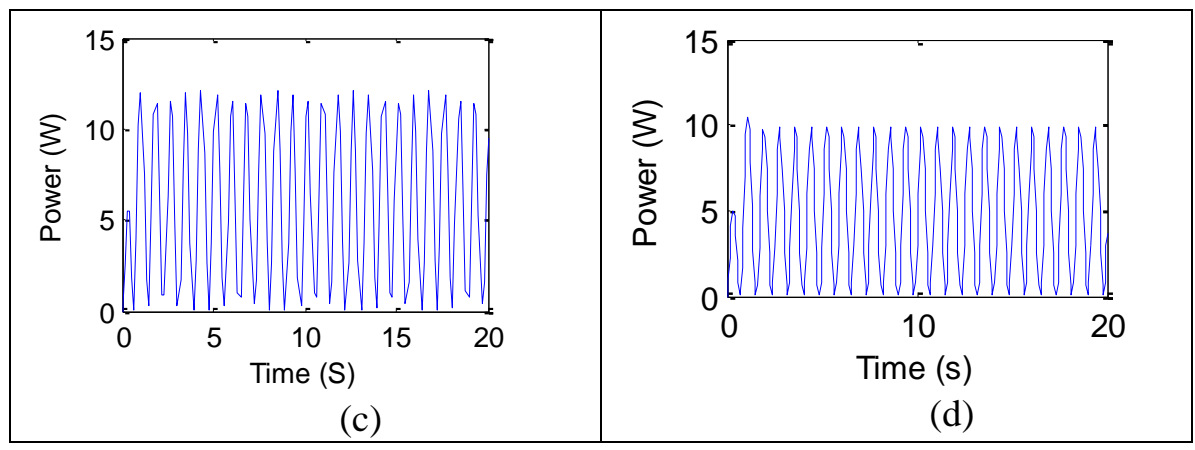

It is observed from Figure 16 that the harvested power is increased when the actuator constant is increased to higher values. The generated power when the actuator constant is $2 K_{a}$ is higher than when it is $K_{a}$ and is larger also when it is $K_{a} / 2$. This shows that increasing the actuator constant value will generate more power from the suspension system for the same input excitation. However when the actuator constant value is increased beyond $2 K_{a}$ the change in the harvested power is not significant. This shows that the actuator constant can be adjusted to generate maximum power for an input excitation frequency.

\section{$5 \quad$ Conclusions}

In this paper, the amount of energy that can be harvested from suspension was investigated using a road simulation rig and the experiments performed on an actual car to estimate the amount of harvested energy for a wide range of excitation frequencies. Theoretical and experimental values of harvested energy were calculated. Experimental evaluation of the energy is performed using vehicle road simulation facilities. Excitation signals in the frequency range of $0.5 \mathrm{~Hz}$ to $20 \mathrm{~Hz}$ were applied to the vehicle. Harvested power was calculated for these input excitation frequencies. Experimental results showed maximum harvested power of $984.4 \mathrm{~W}$ at the highest frequency, which was close to the theoretically calculated value of $1106 \mathrm{~W}$, for each suspension. Application of Regenerative Force Actuators (RFA) was explored for harvesting the vibration energy and controlling the vibration. For a certain range of the actuator constant value, it was shown that the harvested power increased with the actuator constant.

\section{References}

De Silva, C. W., (2007) 'Vibration-Fundamentals and Practice,' Taylor \& Francis/CRC, $2^{\text {nd }} E d$.

Faris, W. F., Ihsan, S. I., Ahmadian, M., (2009) 'A comparative ride performance and dynamic analysis of passive and semi-active suspension systems based on different vehicle models,' Int. J. of Vehicle Noise and Vibration, Vol. 5, No. 1/2, pp. 116 - 140. 
Faris, W. F., BenLahcene, Z., Ihsan, S. I., (2009) 'Analysis of semiactive suspension systems for four-axles off-road vehicle using half model,' Int. J. of Vehicle Noise and Vibration, Vol. 5, No. $1 / 2$, pp. $91-115$.

Goldner R. B., Zerigian, P., and Hull J. R., (2001) 'A preliminary study of energy recovery in vehicles by using regenerative magnetic shock absorbers,' SAE technical paper series, \#2001-01-2071.

Goldner, R. B., and Zerigian, P. (2005) 'Electromagnetic linear generator and shock absorber,' US Patent Specification, 6,952,060 B2.

Graves, K. E., Iovenitti, P. G. and Toncich, D., (2000) 'Electromagnetic regenerative damping in vehicle suspension systems.' International Journal of Vehicle Design, Vol. 24, No.2/3, pp. 182 - 197.

Gupta, A., Jendrzejczyk, J. A., Mulcahy, T. M., and Hull J. R., (2006) 'Design of electromagnetic shock absorbers,' International Journal of Mechanics and Materials in Design, Volume 3, Number 3, pp. 285291.

Ihsan, S. I., Faris, W. F., Ahmadian, M., (2007) 'Dynamics and control policies analysis of semi-active suspension systems using a full-car model,' Int. J. of Vehicle Noise and Vibration, Vol. 3, No. 4, pp. 370 - 405.

Kim, S. S., and Okada, Y., (2002) 'Variable resistance type energy regenerative damper using pulse width modulated step-up chopper,' Journal of Vibration and Acoustics, 124, 110-5.

Khoshnoud F., and de Silva, C. W., (2012) 'Recent advances in MEMS sensor technology - Mechanical Applications,' IEEE Instrumentation and Measurement Magazine, Volume 15, Issue 2, pp. 14 - 24.

Khoshnoud, F., Owhadi, H., de Silva, C. W., Ventura, C. E., and Zhu W., (2011) 'Energy harvesting from ambient vibration with a nanotube based oscillator for remote vibration monitoring,' Proc. of the Canadian Congress of Applied Mechanics, Vancouver, BC, June 2011.

Nakano, K. and Suda., Y., (2004) 'Combined type self-powered active, vibration control of truck cabins,' Vehicle Systems Dynamics, 41, pp. 449-73.

Nakano, K., Suda, Y. and Nakadai, S., (2003) 'Self-powered active vibration control using a single electric actuator,' Journal of Sound and Vibration, 260, pp. 213-35.

Okada, Y., Harada, H., and Suzuki K., (1997) 'Active and regenerativecontrol of an electrodynamic-type suspension,' JSME international journal. Series $C$, Mechanical systems, machine elements and manufacturing, 40(2), pp. 272-278. 
Title

Paz, O. D., (2004) 'Design and performance of electric shock absorber, Master's Thesis, Louisiana State University, USA.

Scruggs, J. T., (2004) 'Structural control using regenerative force actuation networks,' Ph.D. Dissertation, California Institute of Technology, Pasadena, CA, USA.

Scruggs J.T. and Skelton, R.E., (2006) 'Regenerative Tensegrity Structures for Energy Harvesting Applications,' 45th IEEE Conference on Decision and Control, San Diego, pp. 2282-2287.

Stephen, N. G., (2006) 'On energy harvesting from ambient vibration, Journal of Sound and Vibration, 293, pp. 409-425.

Suda, Y., Nakadai, S. and Nakano., K. (1998) 'Hybrid suspension, system with skyhook control and energy regeneration (Development of selfpowered active suspension),' Vehicle System Dynamics, 19, pp. 61934.

Zheng, L. and Baz, A., (2007) 'Control of vehicle suspension using

a Non-linear Energy Sink controller,' Int. J. of Vehicle Noise and

Vibration, Vol. 3, No. 1, pp. 27 - 45.

Zuo, L., Scully, B., Shestani, J. and Zhou, Y., (2010) 'Design and characterization of an electromagnetic energy harvester for vehicle suspensions,' Smart Materials and Structures, 19, 045003 (10pp).

Zuo, L. and Zhang, P., (2012) 'Energy harvesting, ride comfort and road handling of regenerative vehicle suspensions,' ASME Journal of Vibrations and Acoustics, in print. 